\title{
Expériences poétiques d'étudiants en formation initiale en enseignement du français au secondaire québécois
}

\author{
JUDITH ÉMERY-BRUNEAU \\ Université du Québec en Outaouais \\ CAMILLE S. LECLERC \\ Université du Québec en Outaouais
}

\begin{abstract}
Résumé
Quel rapport à la poésie contemporaine les étudiants en formation initiale à l'enseignement du français au secondaire québécois entretiennent-ils? Quelles sont leurs conceptions de la poésie, leur manière de lire un recueil de poésie contemporaine et d'écrire un poème? Nos résultats d'enquêtes montrent le très lourd poids de la tradition scolaire qui pèse sur les étudiants et qui semble les empêcher de se distancier afin d'éviter de reproduire des pratiques plus scolaires qu'expérientielles ou esthétiques. Nous proposons quelques pistes de formation qui leur permettront de vivre des expériences poétiques variées, déstabilisantes, satisfaisantes, fructueuses et complexes afin de les amener à enseigner autrement la poésie.
\end{abstract}

\section{Mots-clés}

poésie, expérience poétique, lecture littéraire, sujet lecteur, enseignement secondaire, formation des enseignants

\section{Introduction}

Qu'est-ce que la poésie? Comment la lire? Comment en écrire? Comment l'enseigner? Si quelques passionnés se réjouissent de lui accorder quelques cours, plusieurs enseignants du secondaire la délaissent ou l'enseignent sans plaisir ni conviction. Cet objet aux formes multiples, appelant les consciences à s'ouvrir à l'impossible, générant des expériences souvent personnelles et parfois collectives, de nature affective, performative, politique, esthétique... transforme. La poésie transforme « une forme de vie par une forme de langage et [...] une forme de langage par une forme de vie, toutes deux inséparablement» (Meschonnic, 2017, p. 58). Si quelques aficionados acceptent volontairement de vivre des expériences poétiques dans leur quotidien et souhaitent en être transformés sans présenter trop de résistance, qu'en est-il lorsque la poésie est travaillée en contexte scolaire? Quelles sont les pratiques des enseignants, obligés par les prescriptions de l'enseigner? Et quels en sont les échos chez les futurs enseignants de français?

Dans cet article, nous tentons de comprendre le rapport à la poésie des futurs enseignants. Notre méthodologie nous a permis de cerner leurs conceptions de la poésie, leur manière de lire un recueil de poésie contemporaine et d'écrire un poème. Les résultats de cette étude de cas montrent le très lourd poids de la tradition scolaire qui pèse sur les étudiants et qui semble les empêcher de se distancier afin d'éviter de reproduire des pratiques plus scolaires qu'expérientielles ou esthétiques. Nous proposons en conclusion quelques pistes de formation qui leur permettront de vivre des expériences 
poétiques variées, déstabilisantes, satisfaisantes, fructueuses et complexes afin de les amener à enseigner autrement la poésie.

Problématique : poésie et formation initiale des enseignants du secondaire

La formation initiale à l'enseignement du français (FIEF) au secondaire québécois en regard de la poésie est marginalisée et déficiente, malgré le fait que les enseignants doivent la travailler avec leurs élèves au cours de leurs pratiques professionnelles, puisqu'il est prescrit, dans les programmes actuels (MÉQ [Ministère de l'Éducation du Québec], 2005; MÉLS [Ministère de l'Éducation, du Loisir et du Sport], 2009, 2011) comme dans les anciens d'ailleurs (Melançon, Moisan et Roy, 1988), de travailler des textes de genres poétiques à chacune des cinq années du secondaire. Or, l'analyse des programmes de FIEF offerts par les neuf universités québécoises montre que seulement trois des neuf programmes proposent un cours disciplinaire obligatoire qui porte spécifiquement sur la poésie ${ }^{1}$ et deux autres programmes offrent un cours où la poésie est l'un des genres étudiés parmi d'autres ${ }^{2}$. En outre, aucun n'offre de cours de didactique axé sur la poésie, cette dernière étant néanmoins évoquée dans quelques descriptifs des cours de didactique de la littérature, de la lecture littéraire ou de la création littéraire, ce qui signifie qu'elle est étudiée, ou non, selon les choix des formateurs et didacticiens. Aussi, si la formation universitaire des futurs enseignants ne leur permet pas de réfléchir à leur rapport à la poésie et à la manière de l'enseigner, il y a fort à parier que leur pratique d'enseignement risque de s'inscrire dans une logique de reproduction, c'est-àdire qu'ils imiteront les pratiques d'enseignement qu'ils ont eux-mêmes connues lorsqu'ils étaient élèves du secondaire ou du cégep (Émery-Bruneau, 2013), n'étant pas suffisamment outillés sur les plans disciplinaire et didactique pour se sentir à l'aise d'accompagner leurs élèves dans la découverte, l'analyse ou la création de poèmes de tout genre. Pourtant, même s'ils souhaitaient reproduire ce qu'ils ont vécu élèves, les récentes recherches sur les corpus enseignés (Dezutter, Larivière, Bergeron \& Morissette, 2007) ou sur les pratiques déclarées d'enseignement de la littérature (Émery-Bruneau, 2014) ont montré que les œuvres complètes lues au secondaire sont des romans ou des pièces de théâtre : aucun recueil de poèmes ne fait partie du corpus obligatoire. En effet, « le genre narratif règne en maitre dans les corpus d'enseignement, et la part accordée à la poésie est minime » (Duval, 2004, p. 42), puisque les enseignants se disent peu à l'aise avec les textes de genre poétique (Duval \& Turcotte, 2007).

La poésie est effectivement souvent négligée à l'école, comparativement aux exercices de grammaire, aux activités de compréhension en lecture ou aux activités de vocabulaire qui sont les trois pratiques hebdomadaires les plus fréquentes dans les classes de français du secondaire québécois (Chartrand \& Lord, 2010, p. 30). Et si la poésie est enseignée, une seule séquence d'enseignement y est consacrée annuellement et «ce sont les mêmes objets enseignés qui reviennent systématiquement (règles de versification, liste de figures de style, poème à forme fixe) quel que soit le texte travaillé (poème classique, contemporain, [slam] ou chanson), que ces objets soient prescrits ou non pour

\footnotetext{
1 Université du Québec à Trois-Rivières (LNG1070: Le langage poétique); Université du Québec à Rimouski (MET10110 : Approches de la poésie); Université du Québec à Montréal (LIT6235 : Histoire et esthétique de la poésie).

${ }^{2}$ Université du Québec en Abitibi-Témiscamingue (LIT1226 : Contes, poésie et chanson); Université Laval (LIT1908 : Lire la poésie et le théâtre).
} 
l'année scolaire où ils [sont] enseignés » (Émery-Bruneau, 2016, p. 133). S'il reste à « inventer » une didactique de la poésie, défend Brillant-Rannou (2010), alors la poésie serait-elle d'abord à démystifier et à démythifier chez les enseignants afin qu'ils se sentent plus à l'aise de la travailler avec leurs élèves? Et comment mieux comprendre leur rapport à la poésie dès leur formation initiale afin de les former plus adéquatement à lire, à écrire, à écouter et à performer oralement des textes de genres poétiques?

Dans une recherche précédente sur le rapport à la lecture littéraire (RLL) des étudiants en FIEF (Émery-Bruneau, 2010), nous avions étudié les dimensions qui marquaient les parcours de ces sujets-lecteurs et leurs conceptions de la littérature. Les résultats provenaient de deux types de données (bilans de savoir et entretiens semidirigés), utilisées pour comprendre de façon globale le RLL; nous soulignions toutefois ne pas avoir été en mesure de comprendre comment le RLL se vit réellement lors d'une ou de diverses expériences de lecture littéraire. Dans les prolongements possibles de cette recherche (p. 370-371), nous notions qu'il faudrait aller au-delà des discours des sujets et observer comment ce « rapport à » se manifeste concrètement. Pour y arriver, une des pistes proposées était de mener une recherche pour comprendre la façon dont des étudiants en FIEF lisent, comprennent et interprètent une œuvre littéraire, notamment à l'aide de réactions écrites et de leurs discours sur ces réactions. Pour la présente contribution, nous avons donc décidé de pallier ce manque, car nous souhaitons comprendre comment la poésie les «interroge» (Siméon, 2015), bouscule leurs conceptions et crée des effets sur leurs expériences de lecture.

Notre travail s'est donc organisé autour des questions suivantes : quelles sont les conceptions de la poésie des étudiants en FIEF au secondaire québécois? Quelles expériences poétiques vivent-ils lorsqu'ils lisent un recueil de poésie contemporaine et comment entrent-ils en dialogue avec le texte et avec le poète? Et à quoi ressemblent leurs poèmes lorsqu'ils sont appelés à en créer un à leur tour? Pour y répondre, trois objectifs ont été ciblés : 1) décrire les conceptions de la poésie d'étudiants en FIEF; 2) analyser leurs expériences poétiques d'un recueil de poésie québécoise contemporaine; 3 ) comprendre leur rapport à la poésie par leurs conceptions de cet objet, leurs lectures créatives et leurs créations de poèmes.

\section{Cadre conceptuel : poésie, expérience poétique et lecture créative}

Chaque lecture d'un poème renouvèle la manière de concevoir la poésie ou, pour citer Brillant-Rannou (2010, p. 30) : «Un poème est pluriel, il jouit d'autant de réalités que de lectures l'actualisant. » Pour ne pas la réduire à une production de sens issu de la combinaison de signes qui la composent (Benveniste, 1969), Meschonnic (2017) montre que la poésie est l'inscription d'un sujet historique dans le langage à savoir que tout ce qui touche le langage le transforme. Pour lui, «il y a une invention du sujet [à la seule condition] que le poème transforme la vie comme la vie transforme le langage, et la poésie » (p. 58). La transformation du sujet par la poésie serait à ce point puissante qu'elle pourrait même sauver le monde, comme le souligne Siméon (2015), puisqu'elle modifie les consciences : elle "n'est pas un communiqué, elle n'informe de rien : elle interroge» (Siméon, 2015, p. 37). Non seulement art du langage, la poésie est «l'aventure d'un sujet engagé tout entier dans une traversée du monde et du langage » Collot (2005, p. 155). La poésie est donc expériences - du langage, de soi, de l'Autre et du monde - car elle fait émerger des sensibilités et des réflexions pour accéder à une 
forme de connaissance « en marge de la pensée » (Bonnefoy, 1998, p. 110). Elle ouvre la voie à cette diversité de lectures intime, personnelle, sociale, éthique et même politique par lesquelles le rapport à la poésie des sujets-lecteurs se transforme. Bref, « le poème devient l'objet unique du lecteur, non pas dans le sens égotique et exclusif de la propriété, mais dans celui d'une expérience » (Brillant-Rannou, 2010, p. 16).

Si les sujets-lecteurs se transforment personnellement - et didactiquement, dans le cas des enseignants - à chaque expérience poétique, à chaque lecture créative ou débat interprétatif, leur rapport à la poésie s'en trouve aussi transformé. Nous pensons en outre que leur aisance à lire des poèmes et à en parler sera bonifiée. En effet, « la connaissance n'advient et ne vaut que chevillée à l'expérience, et [le] savoir se construit sur les vases d'un vécu effectif, personnel, voire sensoriel » (Brillant-Rannou, 2016, p. 87). Mais pour $\mathrm{y}$ arriver, il importe, dans la formation des lecteurs de poésie, de les amener à prendre conscience de leur lecture créative et à mieux comprendre leurs gestes de lecteurs (lectures tabulaires ou linéaires, soulignage/surlignage, annotations dans les marges, sélection d'extraits, consignation d'extraits dans un carnet, etc.) et leur activité fictionnalisante (Langlade \& Fourtanier, 2007).

À cet effet, les cinq processus subjectifs d'activité fictionnalisante du sujet lecteur peuvent s'observer dans toute lecture créative (Langlade \& Fourtanier, 2007) : la concrétisation imageante, c'est-à-dire les images mentales ou «propres configurations internes » produites par le lecteur, en complément de l'œuvre; la cohérence mimétique, soit la construction de la cohérence causale à la lumière des attentes de mimétisme du sujet-lecteur; l'activation fantasmatique, c'est-à-dire les fascinations, les répulsions, les dégouts, etc. à l'égard d'éléments du poème; les réactions axiologiques, soit les jugements moraux du sujet en référence à son propre système de valeurs symboliques, la compréhension ou la perplexité que lui inspirent des extraits du recueil et qui jouent un rôle de premier plan dans l'activité interprétative; le rapport esthétique, renvoyant à la forme, à la stéréotypie, aux genres, etc. Car si la lecture littéraire est définie depuis Dufays (1994), lui-même inspiré de Picard (1986), comme un va-et-vient dialectique entre une lecture participative empreinte de réactions spontanées et une lecture distanciée réalisée de façon plus analytique et intellectualisée, ce sont les opérations de lecture (compréhension, interprétation, réactions affectives, appréciation critique), croisées aux gestes des sujets-lecteurs et aux processus subjectifs de leur activité fictionnalisante, qui nous permettent de cerner ce que saisit le sujet-lecteur, ce qu'il propose comme significations diverses, ce qu'il préfèrerait voir dans le texte, etc.

Somme toute, pour la didactique, ce qui nous intéresse n'est donc pas que l'origine d'une lecture subjective, mais plutôt les effets de la subjectivité et de la réflexivité sur l'expérience de lecture, soit cette lecture créative que Langlade (2012, p. 50) définit comme «la part originale, nouvelle, inattendue de lisible qu'elle met en évidence ou qu'elle crée dans l'œuvre ", à savoir la reconfiguration du texte, sa réécriture, voire la création d'un poème du lecteur.

\section{Méthodologie}

Pour cette étude de cas, nous avons travaillé à partir d'un échantillon de convenance constitué d'un corpus d'une douzaine de textes, de six à huit pages chacun, produits par 12 étudiants en FIEF au secondaire québécois. Ils devaient répondre à cinq questions, lesquelles les invitaient notamment à décrire leurs conceptions de la poésie et 
leur expérience poétique de la lecture d'un recueil de poésie contemporaine, mais aussi à formuler des questions qu'ils souhaitaient adresser à la poète ainsi qu'à écrire un poème.

\section{Lecture d'un recueil de poésie contemporaine et réponses à un questionnaire écrit}

Dans le cadre d'un cours offert lors de la deuxième des quatre années de formation universitaire en enseignement du français au secondaire, ces étudiants ont été invités à répondre par écrit à cinq questions (voir tableau 1). Ils avaient trois semaines pour lire un recueil de poésie québécoise contemporaine et pour répondre à chacune de ces questions (en dehors de la classe) en une page environ. À ce moment de leur parcours universitaire, ces étudiants avaient globalement suivi deux cours de littérature, deux cours de grammaire, six cours en fondements de l'éducation et un premier stage de formation pratique. Les questions leur ont été posées à la première séance de leur premier cours de didactique de la littérature et n'ont pas fait l'objet d'une discussion en classe. Le tableau suivant présente les cinq questions, accompagnées des angles de la recherche liés à chacune d'elles.

Tableau 1

Questions posées aux étudiants en FIEF

\begin{tabular}{|l|l|}
\hline Angles de la recherche & \multicolumn{1}{c|}{ Questions posées } \\
\hline $\begin{array}{l}\text { Conceptions de la } \\
\text { poésie }\end{array}$ & $\begin{array}{l}\text { 1. Demandez-vous ce qu'est la poésie pour vous et expliquez-le. Vous } \\
\text { pouvez vous appuyer sur des auteurs ou des textes scientifiques, si vous } \\
\text { le souhaitez. }\end{array}$ \\
\hline \multirow{5}{*}{ Expérience poétique } & $\begin{array}{l}\text { 2. Lisez le recueil de poésie Le ciel comme passage, de la manière qui } \\
\text { vous plaira, puis formulez un commentaire sur votre expérience de } \\
\text { lecture de ce recueil (réactions, émotions, réflexions, analyse, etc.). }\end{array}$ \\
\cline { 2 - 3 } Lectures créatives & $\begin{array}{l}\text { 3. Choisissez un poème du recueil (précisez la page). Expliquez pourquoi } \\
\text { vous l'avez choisi. Expliquez ce qu'il vous fait vivre. Expliquez ce que } \\
\text { vous en comprenez. }\end{array}$ \\
\hline \multirow{5}{*}{ Écriture d'un poème } & $\begin{array}{l}\text { f. Formulez trois questions que vous aimeriez poser à la poète Isabelle } \\
\text { Duval, lesquelles vous permettraient de mieux comprendre son recueil, } \\
\text { de connaitre son processus de création ou de partager votre point de vue } \\
\text { sur sa poésie, à votre guise. Utilisez des exemples pour éclairer vos } \\
\text { propos. }\end{array}$ \\
\hline $\begin{array}{l}\text { 5. Et si vous deviez réagir à un poème de ce recueil (choisissez-en un } \\
\text { différent de celui de la question 3) par la création d'un poème, à quoi } \\
\text { ressemblerait votre création? Écrivez ce poème puis expliquez votre } \\
\text { processus de création. }\end{array}$ \\
\hline
\end{tabular}

Le recueil de poésie choisi s'intitule Le ciel comme passage, de la poète québécoise Isabelle Duval, paru aux éditions Les Herbes rouges en 2014. Il s'agit d'un recueil de 75 pages, divisé en trois parties. Les poèmes sont courts (une demi-page) et abordent des sujets et thèmes introspectifs : l'existence, la spiritualité, les relations intimes, les espaces, les passages. Sur le site web ${ }^{3}$ du même nom que le recueil, Isabelle Duval affirme que son écriture poétique est un « voyage des voyages, [une] traversée des traversées. » Elle y parle de sa démarche en affirmant que « ses textes sont marqués par la

\footnotetext{
${ }^{3}$ Duval, I. (n.d.). Le ciel comme passage. Récupéré à : https://lecielcommepassage.com/demarche_isabelle_duval/
} 
quête de la transcendance, le désir de se "rapailler", de s'inscrire dans l'expérience du monde le plus justement possible, pour qu'émerge une parole vraie. » Avec son recueil, c'est un véritable dialogue qu'elle ouvre avec ses lecteurs :

«Comment amener le lecteur ou le spectateur à vivre une expérience poétique? À connaître et à éprouver de l'intérieur les enjeux posés par la poésie? Telles sont les questions qui orientent mon travail, que je situe dans le cadre d'interrogations plus vastes sur les liens qui unissent l'homme au sacré, à la mémoire, aux paysages auxquels il se confronte et qui le construisent. »

Ce recueil de poésie a été choisi en raison des critères suivants : il s'agit d'un recueil de poésie écrit par une poète québécoise de la relève; il s'agit de poèmes résistants (Tauveron, 1999), car ils sont polysémiques, ce qui exige des lecteurs un travail d'analyse approfondie générant une diversité d'interprétations possibles; enfin, c'est un recueil de poésie contemporaine qui s'est distingué en recevant une reconnaissance du public, puisqu'il a été sélectionné dans la catégorie « coups de cœur des lecteurs » du prestigieux Prix de poésie Radio-Canada en 2015.

\section{L'analyse de contenu des questionnaires écrits}

Pour l'ensemble des données issues des réponses au questionnaire, une analyse de contenu a été réalisée (L'Écuyer, 1990; Bardin, 1993). Cette méthode de codification et de classification nous a permis de voir émerger les conceptions de la poésie, les expériences poétiques et les lectures créatives de ce recueil par ces sujets-lecteurs. L'ensemble des données a fait l'objet d'une triple analyse : la première a mené à la constitution des catégories émergentes pour traiter chaque question, la deuxième a permis de valider les catégories en soumettant le quart des données à une analyse intra et intercodeurs (taux d'accord de 85\%) et la troisième a été faite afin de réviser et d'uniformiser les analyses de l'ensemble des données. Les catégories apparaissent dans la section des résultats (voir les tableaux 2,3,4 et 5). Ces derniers ont été interprétés à l'aide de notre cadre théorique, mais aussi mis en dialogues avec d'autres recherches. Rappelons, à l'instar de Brillant-Rannou (2010, p. 19), que «[t]oute trace de lecture est à étudier en tant que texte, avec sa polysémie potentielle et une marge d'interprétation. Si chaque lecture est celle d'une personne, c'est bien la lecture qui est visée par l'analyse et non le sujet historique qui la vit. »

\section{Résultats}

Les résultats sont présentés en trois parties: nous décrivons d'abord les conceptions de la poésie émergeant des discours écrits des étudiants (question 1), suivies de leurs expériences poétiques illustrées à l'aide de lectures créatives (question 4), puis des écritures de la réception du recueil de poésie (question 5). Nous ne décrivons pas dans cet article l'ensemble des résultats issus des questions 2 et 3, mais nous nous y référons à plusieurs égards pour éclairer nos analyses des questions 4 et 5 .

Conceptions de la poésie

Le tableau 2 présente la liste des catégories émergentes puis validées que nous 
avons utilisées pour coder et classer les unités de sens de la question 1.

Tableau 2

Résultats de l'analyse de contenu (conceptions de la poésie : question 1)

\begin{tabular}{|l|r|}
\hline \multicolumn{1}{|c|}{ Conceptions de la poésie } & n= \\
\hline Auteur (expression de soi, de ses émotions, de ses sentiments) & 25 \\
\hline Lecteur (émotions, réactions, analyse) & 25 \\
\hline Forme (fixe ou libre), structure, versification & 16 \\
\hline Genre et caractéristiques textuelles & 12 \\
\hline Vision du monde, point de vue & 11 \\
\hline Histoire littéraire (auteur, contexte de production) & 11 \\
\hline Travail de création, processus de création, imaginaire & 7 \\
\hline Sens, connotation, valeurs, message & 6 \\
\hline Rythmes et sonorités (rimes, répétition, etc.) & 5 \\
\hline Images & 3 \\
\hline Figures de style, procédés d'écriture & 3 \\
\hline Travail sur la langue (registre de langue, ponctuation, etc.) & 2 \\
\hline Thèmes/champs lexicaux & 2 \\
\hline Nombre total d'unités de sens & $\mathbf{1 2 8}$ \\
\hline
\end{tabular}

L'analyse de contenu a permis de cerner dans le discours des étudiants de notre échantillon trois principales conceptions de la poésie: la première est tournée vers l'intention de l'auteur, la seconde porte sur l'expérience de lecture, la troisième renvoie aux formes et structures des textes.

Plus du tiers des unités de sens codés (50/128) réfèrent en effet aux catégories auteur et lecteur, car la poésie renvoie pour eux à un objet lié aux émotions, permettant l'expression de soi à travers l'écriture poétique $(\mathrm{n}=25)$ ou faisant vivre des émotions au lecteur $(n=25)$. Il s'agit aussi pour eux de poèmes organisés, structurés, adoptant des formes généralement fixes $(n=16)$. Puis, dans une moindre mesure, d'autres adoptent un point de vue plus représentationaliste, considérant que les poèmes évoquent une certaine vision du monde ou un point de vue particulier sur le monde $(n=11)$ ou alors, ils décrivent la poésie dans une perspective historique, soulignant les grands auteurs et courants littéraires $(n=11)$. En revanche, le travail sur la langue $(n=2)$ et les procédés d'écriture $(n=3)$ sont des aspects très peu présents de leurs discours, ce qui nous fait dire que ces étudiants ne semblent pas considérer la poésie comme un art du langage. Ajoutons en terminant que trois étudiants ont appuyé leurs propos sur ceux énoncés par des poètes (Émile Nelligan, Victor Hugo) souvent enseignés au secondaire québécois et quatre étudiants se sont appuyés sur un ouvrage didactique (Chartrand, Émery-Bruneau \& Sénéchal, 2015), utilisé dans les cours universitaires en FIEF, pour préciser leur définition des genres poétiques (poème lyrique, poème engagé).

\section{Écrire la poésie pour exprimer son intériorité.}

$\mathrm{Si}$ les trois quarts des participants affirment que la poésie renvoie à l'expression de soi de l'auteur, de ses émotions et de ses sentiments, cette idée marque cependant leur 
propos de différentes manières : certains n'y réfèrent qu'une fois, tandis que d'autres y réfèrent quatre ou six fois en une dizaine de lignes. Pour eux, les poèmes dévoileraient une partie de l'intimité ou de la psyché de l'auteur et illustreraient aussi un rapport à soi, aux autres et au monde : «La poésie peut nous permettre de nous révéler à nous-mêmes et aux autres » $(\mathrm{E} 1,2)$. Dans cette perspective d'influence psychocritique, l'auteur «ne cherche pas nécessairement à se faire comprendre, ni à passer un message » $(\mathrm{E} 6,2)$ parce «[qu'i]l peut aussi écrire simplement pour lui-même» $(E 11,1)$, pour mieux se comprendre ou illustrer sa vision du monde : «la poète [...] fait découvrir son univers parallèle » $(\mathrm{E} 9,1)$. Les poèmes donnent, à leur avis, accès à l'intimité de l'auteur et à ses émotions, voire à son inconscient : « la poésie est bien difficile à définir, mais pour moi, elle est vivante et est intime » $(\mathrm{E} 5,4)$, « la poésie a quelque chose d'intime : c'est pour moi une façon d'attribuer à un texte que j'écris une valeur sentimentale » $(E 7,1)$.

La notion de la liberté est fréquemment évoquée par les étudiants pour parler du poète et certains affirment que la poésie permet à l'auteur de vivre sa catharsis : «la poésie est, selon moi, la forme de texte littéraire dans laquelle l'auteur peut le plus librement laisser transparaitre ses émotions » $(E 9,1)$. En outre, les formes fixes associées à la poésie classique sont critiquées par un étudiant qui mentionne qu'elles « découragent souvent les écrivains d'opter pour ce genre de texte qui, pourtant, est excellent pour exprimer des émotions intérieures et pour créer l'univers de notre choix (abstrait, fantastique, réaliste, romantique, etc.) grâce à notre écriture » $(\mathrm{E} 10,2)$. Il semble donc qu'une conception influencée par la psychocritique est assez partagée par ces étudiants : la poésie serait pour eux un vecteur facilitant l'expression de soi, de ses émotions, de son intériorité ou, comme le défendait Mauron (1963), l'œuvre est le fruit d'un autre moi que celui de la vie sociale.

\section{Lire la poésie : un défi s'adressant à un lecteur modèle.}

Lorsqu'ils mentionnent que la poésie permet aux lecteurs de vivre des émotions, les étudiants précisent qu'elles peuvent être vécues de façon affective, en termes de réactions spontanées ou réflexives, à la suite d'une analyse satisfaisante sur le plan intellectuel qui leur permet d'apprécier l'esthétique du poème. Dans les deux cas, c'est selon eux au lecteur d'achever le poème, car «[le poème], une fois sorti de la plume du poète, ne lui appartient plus » $(\mathrm{E} 1,1)$.

Dix des 12 participants font d'ailleurs référence au lecteur à la question 1 . Ils affirment néanmoins que la poésie est un genre difficile à lire et à comprendre, notamment en raison de l'absence de diégèse pouvant faciliter la compréhension littérale ou de leur remise en question de leurs compétences lectorales : "la compréhension de poèmes s'est toujours avérée complexe, car il est difficile de savoir comment s'y prendre » $(\mathrm{E} 11,2)$; «Se plonger dans un recueil de poésie quand on n'est accoutumé qu'aux stéréotypes qui y sont liés n'est pas chose facile» $(\mathrm{E} 9,7)$; «il est ainsi très difficile pour le lecteur de cerner ce que l'auteur tentait de véhiculer exactement »(E11, 1-2). Un autre participant conclut même en rappelant que la poésie ne fait pas partie de ses pratiques personnelles de lecture parce qu'il la juge trop difficile d'accès : « [la poésie est] un genre littéraire que j'écarte habituellement, car je le trouve difficilement accessible » (E6, 7).

Le défi lectoral que présente la poésie et les difficultés de compréhension qu'elle pose sont répandus dans les conceptions des étudiants en raison de leur insécurité à 
accepter la dimension polysémique des poèmes et de leur manque de repères pour arriver à les interpréter de façon satisfaisante. Comme le précise un étudiant, « le lecteur ne peut qu'utiliser ses propres connaissances pour "vivre" un poème » $(\mathrm{E} 11,1-2)$. Sa lecture fait donc émerger des «interprétations différentes selon le bagage du lecteur, les différentes lectures du même poème, l'analyse qu'on en fait, selon les commentaires des autres lecteurs qui influencent les perceptions du nouveau lecteur ou du "relecteur" » $(\mathrm{E} 5,2)$. Si la contribution des ressources subjectives du lecteur, au sens de Sauvaire (2013), sert souvent à justifier la dimension subjective et personnelle de l'interprétation du poème, le sens du texte poétique est jugé flou par les étudiants, voire hermétique. Des énoncés tels que « le poète ne veut pas énoncer clairement son propos, sinon ce ne serait pas un poème qu'il écrirait » $(E 11,1)$ ou «L'univers du poème est très vague ce qui laisse autant de liberté à l'interprétation du lecteur » $(\mathrm{E} 12,1)$ montrent d'autant plus que cette confusion autour du sens et des significations est une préoccupation manifestement non négligeable chez les étudiants en FIEF qui affirment du même coup, et de façon à peine voilée, se sentir peu outillés et peu expérimentés pour comprendre la poésie.

\section{La poésie : une question de formes.}

Bien que moins représentées que les conceptions associées à l'auteur et au lecteur, les caractéristiques associées à la forme du poème marquent aussi le discours des étudiants. La structure du texte (texte en prose, organisation en strophes) ainsi que la versification sont mentionnées par tous les étudiants, sauf un. L'habitus scolaire (Bourdieu, 1980), par lequel ils ont appris à disséquer des poèmes est bien ancré dans le discours de certains étudiants: «les caractéristiques principales sont l'obligation d'instaurer des rimes, la division du contenu sous forme de vers et de strophes, les formes fixes (rondeau, ballade, sonnet), les formes "libres" (vers libres, verset, prose), la construction des vers en alexandrin, la présence des sentiments, l'usage de figures de style, etc. » $(E 10,2)$. L'un des participants attribue même sa conception stéréotypée du poème à son parcours scolaire marqué d'une rhétorique classique : «[...] j'ai longtemps pensé que la poésie devait nécessairement être organisée en vers symétriques contenant des rimes. Cette conception stéréotypée a pris naissance à l'école, dans les classes de français » $(\mathrm{E} 8,1)$. Cependant, la prose et la poésie contemporaine semblent aussi s'être taillées une place dans leur vision de la poésie : «Un poème ne doit pas nécessairement contenir des rimes, un titre et un certain nombre de syllabes par vers » $(\mathrm{E} 11,2)$; « Il n'y a pas de structure propre à la poésie » (E5, 1-2). Leurs conceptions sans doute forgées pendant leur scolarité sont ainsi marquées par le formalisme, mais aussi par l'approche historique de la poésie, puisque certains mentionnent les principaux courants littéraires et poètes marquants en rappelant que la poésie s'est graduellement libérée des contraintes qui lui ont été associées.

\section{Expériences poétiques et dialogue avec la poète : l'interprétation avant tout}

Bien que les étudiants aient mentionné que pour eux lire de la poésie n'était pas une activité familière et présentait même plusieurs défis de compréhension, nombreux ont été ceux qui ont néanmoins avancé plusieurs interprétations du recueil de poésie (question 2) ou du poème qu'ils ont préféré (question 3), car les deux tiers des unités de sens analysées (131/199) portaient sur cette opération de lecture. 
Tableau 3

Résultats de l'analyse de contenu (opérations de lecture : questions 2 et 3)

\begin{tabular}{|l|r|}
\hline \multicolumn{1}{|c|}{ Opérations de lecture } & $\mathbf{n =}$ \\
\hline Analyse des significations possibles, interprétation & $\mathbf{1 3 1}$ \\
\hline Réactions subjectives, personnelles, affectives & 25 \\
\hline Appréciation, jugement, critique & 23 \\
\hline Compréhension, explication littérale du sens & 20 \\
\hline Nombre total d'unités de sens & $\mathbf{1 9 9}$ \\
\hline
\end{tabular}

Il semble d'ailleurs que leur expérience poétique s'inscrit d'abord dans une lecture analytique des textes, parfois appuyée sur leur culture première, et visant à faire ressortir une ou des interprétations possibles. De plus, malgré l'invitation à exprimer leur subjectivité (question 3) ainsi que leurs réactions affectives $(n=25)$ et axiologiques $(n=23)$, ces opérations ne représentent pas plus qu'un quart de leurs opérations de lecture. Ce besoin de vouloir saisir les significations de certains passages des poèmes ou d'en connaitre les interprétations possibles se confirme lorsque les étudiants formulent des questions adressées à la poète. À cet effet, les résultats inscrits dans le tableau 4 présentent les sujets sur lesquels les étudiants ont ouvert le dialogue avec la poète. Nous décrivons les résultats qui se démarquent dans les lignes qui suivent.

\section{Tableau 4}

Résultats de l'analyse de contenu (questions à la poète : question 4)

\begin{tabular}{|l|r|}
\hline \multicolumn{1}{|c|}{ Sujets ou thèmes des questions posées } & $\mathbf{n =}$ \\
\hline \multicolumn{1}{|c|}{ Significations/interprétations } & $\mathbf{1 4}$ \\
\hline signification d'un ou de plusieurs passages & 12 \\
\hline signification d'un thème & 2 \\
\hline Processus de création de la poète & $\mathbf{1 4}$ \\
\hline genèse, inspiration, intention de l'auteur & 7 \\
\hline processus d'écriture & 7 \\
\hline \multicolumn{1}{|c|}{ Forme des poèmes } & $\mathbf{4}$ \\
\hline prose, représentation visuelle & 4 \\
\hline Finalité de l'écriture pour la poète & $\mathbf{2}$ \\
\hline catharsis Appréciation & 1 \\
\hline publication & 1 \\
\hline \multicolumn{2}{|c|}{ Appé } \\
\hline écriture - hermétique & $\mathbf{2}$ \\
\hline question/réflexion générale & 1 \\
\hline Nombre de questions posées à la poète & $\mathbf{3 6}$ \\
\hline
\end{tabular}

Dans les 36 questions posées à la poète, 14 d'entre elles avaient pour but de l'interroger sur les interprétations de ses textes. Pour ce faire, ils ont procédé de deux 
façons : soit en citant des passages, soit en la questionnant sur des thèmes qu'ils ont identifiés. Toutes les questions de cet ordre vont dans le même sens, par exemple :

«Dans votre poème à la page 21 , je m'interroge sur la signification des deux dernières strophes. "La route parle pour nous - une langue fragile / que nous ne comprenons pas toujours. // Il n'y a qu'elle pour nous tenir la main / En nous crachant au visage." Que voulez-vous dire par là? Pourquoi la route nous crache-t-elle au visage? Cela signifie-t-il que la vie nous garde bien des surprises aussi bien positives que négatives? » (E2)

«Chacun de vos poèmes inclut le pronom "nous". Pourquoi avoir opté pour ce pronom? Est-ce qu'il vous inclut? Qui tente-til de rejoindre? De décrire? D’inclure? » (E10).

Le deuxième sujet abordé par les étudiants concerne le processus de création de la poète, car 14 des 36 questions portaient sur cet aspect. Il semble que les étudiants cherchent à démystifier d'où provient l'inspiration de la poète. Les questions portent sur des aspects précis des poèmes, comme les figures de style employées : "Vos poèmes regorgent de figures de style (personnifications, métaphores, etc.) [...]. Comment faitesvous pour créer ces images fortes, mais invraisemblables? Quelle est ou quelle a été votre démarche d'écriture? »(E6). Elles sont aussi posées de façon beaucoup plus directe : «Pouvez-vous nous expliquer votre processus de création?» (E1). Les étudiants en FIEF sont curieux de connaitre comment une poète en vient à écrire de la poésie et d'où lui vient cette fascinante inspiration : "Comment commencez-vous à écrire vos poèmes? Partez-vous d'une réflexion, d'une pensée ou bien d'une expérience personnelle vécue? Êtes-vous davantage inspirée à écrire dans un lieu particulier (dans la nature, dans un café, etc.)? »(E2). Ils veulent comprendre comment elle amorce son écriture : «Pourquoi le ciel est-il si important pour vous? Est-il votre source d'inspiration principale? »(E9). Ils veulent aussi mieux connaitre les motivations plus intimes de la poète : « Est-ce un évènement de votre vie qui a provoqué la rédaction de ce recueil? 》 (E12), «Quels sentiments vous traversaient-ils lorsque vous l'avez écrit?» (E9). Ils souhaitent également savoir comment s'y prend concrètement la poète pour écrire ses poèmes afin de mieux connaitre son processus d'écriture. Les questions portent alors sur les moments qu'elle consacre à l'écriture - «Avez-vous une routine d'écriture ou de processus de création? Par exemple, essayez-vous d'écrire un poème dans un temps donné? Est-ce qu'il y a un temps de la journée que vous préférez? »(E11) - ou sur la planification de son écriture : «Réfléchissez-vous beaucoup avant et pendant l'écriture d'un poème, de sorte que vous saviez déjà comment le rédiger, ou bien écrivez-vous plutôt de façon instinctive? » (E8). D'ailleurs, l'instinct semble pour l'un (E8) la clé de réception comme de production de poèmes : «Quand la poésie est mystérieuse, elle confère des émotions plus authentiques. J'ai donc [...] lu le recueil [plus] instinctivement que rationnellement. »

\section{Réagir à un poème par la création d'un poème de lecteur}

La dernière question visait à saisir le rapport à la lecture des étudiants par l'écriture d'un poème exprimant leurs réactions à un poème qu'ils ont choisi dans le 
recueil d'Isabelle Duval. Ils ont aussi décrit leur processus de création. Il s'avère que la grande majorité d'entre eux (10/12) ont écrit «à la manière de... », c'est-à-dire qu'ils ont procédé à des transformations hypertextuelles (Genette, 1982) de l'hypotexte choisi, pratique relativement courante dans les classes. Et pour y arriver, tous disent avoir d'abord analysé le style de l'auteur et tenté de le reproduire, d'y faire allusion ou de transposer son univers poétique à un univers qui leur est plus familier.

\section{Les transformations hypertextuelles sous le joug de l'imitation.}

En reprenant la typologie suggérée par Louichon (2017), dont certaines catégories proviennent initialement de Genette (1982), nous avons constaté que 10 des 12 créations sont des hypertextes tentant d'imiter le style de l'auteur; une création est une transfiction, car on met davantage l'accent sur la dimension diégétique que textuelle, «l'hypertextualité [étant] une relation d'imitation et de transformation entre textes; la transfictionnalité, une relation de migration (avec la modification qui en résulte presque immanquablement) de données diégétiques » (Saint-Gelais, 2011, p. 10-11); et une autre création est une allusion, c'est-à-dire que « le texte n'est pas énoncé, mais il est présent, nécessairement présent »(Louichon, 2017, p. 24).

Les transformations hypertextuelles qui se rapprochent d'une écriture d'imitation s'observent sous plusieurs caractéristiques textuelles reprises de l'hypotexte : l'absence de titre, la reprise d'un des quatre thèmes dominants dans le recueil (existence, passage, voyage, corps) et de leur champ sémantique, les figures de style récurrentes (métaphore, personnification, énumération), le système énonciatif (nous) manifestant sa relation à l'autre et au monde, le système des temps verbaux (présent), la disposition particulière du texte dans l'espace de la page (saut de ligne, alinéas, enjambement). Par exemple, les textes de E4 et de E10 illustrent cette écriture d'imitation :

Tableau 5

Exemples de transformations hypertextuelles

\begin{tabular}{|l|l|}
\hline Début du poème d'Isabelle Duval (p.69) & \multicolumn{1}{c|}{ Début du poème de E4 } \\
\hline $\begin{array}{l}\text { Pendant que la clarté s'enroule sur elle-même, } \\
\text { nous reprenons le chemin du ciel intérieur. }\end{array}$ & $\begin{array}{l}\text { Au moment où le crépuscule recouvre les derniers } \\
\text { rayons du jour, } \\
\text { Nous empruntons sans détour le droit chemin } \\
\text { Qui mène à l'intérieur }\end{array}$ \\
\hline Début du poème d'Isabelle Duval (p.20) & \multicolumn{1}{c|}{ Début du poème de E10 } \\
\hline $\begin{array}{l}\text { À travers le mouvement des foules, } \\
\text { l'âme est un composé d'histoires, un univers } \\
\text { tactile. }\end{array}$ & $\begin{array}{l}\text { À travers nos rencontres } \\
\text { se forge en nous une muraille de sentiments. }\end{array}$ \\
\hline
\end{tabular}

Mais comment les étudiants décrivent-ils leur processus d'écriture?

Des écritures expliquées par le souci de bien imiter.

Tous les étudiants affirment avoir analysé le poème qu'ils ont choisi avant de commencer à créer leur propre poème afin de s'assurer d'en reprendre les principales caractéristiques. Même si la consigne ne les invitait pas à écrire « à la manière de... », ils ont spontanément fait ce choix, comme le mentionnent E3 et E4, dont le processus de 
création est représentatif des autres cas, «j'ai seulement retiré les aspects importants du poème et j'ai, par la suite, continué avec ces idées » (E3), «j'ai imité le style et la structure des phrases construites par la poète Isabelle Duval. J'ai tenté de la paraphraser par endroits, et j'ai fait des ajouts personnels. » (E4). D'ailleurs, leur façon de faire se rapproche d'un processus très scolaire, de l'analyse au brouillon avant d'arriver à la version finale, comme nous l'observons dans le discours de E10 :

«Je me suis fait une compréhension de l'œuvre initiale et ensuite [...] j'ai composé, biffé, recréé et à nouveau effacé. [...] En outre, j'ai créé mon poème selon les mêmes normes d'écriture que celles utilisées dans le poème d'Isabelle Duval, soit aucune rime, aucune continuité dans le nombre de syllabes et aucun titre $[\ldots]$ j'ai utilisé quelques figures de style, comme la personnification, l'allégorie ou encore la comparaison. [...] Finalement, j'ai laissé libre cours à mon imagination et à ma créativité afin de créer un texte qui répondait à des critères d'écriture poétique ».

L'analyse de notre corpus montre que les étudiants s'inscrivent davantage dans une posture analytique que créatrice, car comme l'a souligné Le Goff (2006: 254), «la démarche d'imitation consiste d'abord à restituer à travers l'écriture les significations du portrait dégagées dans le temps de la lecture analytique ».

\section{Interprétation des résultats}

Malgré un nombre limité de données, puisque nous avons réalisé une étude de cas, les résultats obtenus nous permettent de voir le poids de la tradition scolaire dans le rapport à la poésie des étudiants en FIEF, car l'école doit constituer pour plusieurs d'entre eux leur seul contact avec la poésie.

Pour ce qui concerne leurs conceptions, elles sont marquées d'un héritage de la rhétorique scolaire classique. Les résultats confirment aussi que la poésie ne fait pas partie de leur vie: selon les sujets interrogés, elle s'adresse à un lecteur modèle ou expérimenté, capable d'interpréter les textes les plus complexes ou bien elle est un outil d'expression de soi pour les poètes. La poésie semble donc leur être extérieure, elle ne fait pas partie de leur capital culturel et langagier (Bourdieu, 1979), non seulement parce qu'ils n'en lisent pas ou n'en écrivent pas, mais parce qu'on leur en a peu enseigné sinon à partir des figures de style et des formes fixes (Émery-Bruneau, 2016) ou par l'écriture d'imitation, exercice ayant marqué l'enseignement de la littérature depuis des décennies en France (Le Goff, 2006) et en Belgique (Dufays, 1994), comme au Québec (Melançon, Moisan \& Roy, 1988). Le poids de la forme scolaire pèse donc sur leurs conceptions, puisque leurs expériences poétiques s'inscrivent surtout dans une lecture distanciée, analytique et interprétative des textes, contrairement à leur lecture de textes narratifs par exemple, « la majorité des lycéens déclarent emprunter la lecture de type identificatoire, le "playing" (Picard, 1986) pour aborder le roman, tandis que le "game", la lecture distanciée, est plus spontanément adoptée pour la poésie » (Brillant-Rannou, 2016, p. 91). Alors que nos précédents résultats (Émery-Bruneau, 2010) montraient que la lecture participative était plus dominante dans le rapport à la lecture littéraire des étudiants en FIEF, il s'avère qu'en resserrant précisément les analyses sur la lecture d'un recueil de 
poésie, une nuance s'impose: la poésie ne semble pas un langage qu'ils se sont appropriés au point de se sentir suffisamment à l'aise pour en faire une lecture créative, subjective ou même délirante (Bayard, 1998). Elle demeure encore un objet à expliquer plutôt qu'à expérimenter.

Quant au dialogue qu'ils ouvrent avec la poète, près de la moitié des questions visent à mieux comprendre ou interpréter ses poèmes. Sans gêne, ils lui demandent ce que signifient ses poèmes, comme si, d'un côté, ils se sentaient mal à l'aise à l'idée de lui présenter leurs propres interprétations, voire pour certains, incompétents pour le faire. Procéder de la sorte leur permet, certes, d'illustrer leurs besoins de bonifier leur compréhension et leurs interprétations, comme si pour eux la poésie devait seulement être analysée, comme s'ils ne se sentaient pas autorisés à l'expérimenter subjectivement, axiologiquement, émotivement, sensoriellement... faute de connaissances suffisantes et à défaut d'assumer pleinement - et consciemment - leur subjectivité, inhérente au processus interprétatif. Vivre des expériences poétiques variées semble encore être essentiellement réservés aux poètes, car cette pratique expose les sujets à «introduire dans l'analyse une part de subjectivité »(Collot, 2005, p. 155), ce que les étudiants en FIEF n'ont pas appris à faire parce que, d'un côté, ils n'ont pas de pratiques personnelles de poésie et, de l'autre, parce que l'école, avec ses besoins d'enseigner et de faire apprendre des notions observables et mesurables, avec la démultiplication des objets à travailler en classe, n'a pas dégagé un espace pour faire enseigner ainsi la poésie.

N'ayant eux-mêmes pas ou peu expérimenté l'écriture de textes poétiques, malgré qu'elle fasse partie des programmes du secondaire depuis 1995, les étudiants ont posé beaucoup de questions portant sur les origines de l'inspiration de la poète et sur son processus d'écriture, ce qui s'explique par leur besoin de déconstruire le mythe de l'auteur et de démystifier le processus de création. Les questions qu'ils ont posées à la poète nous permettent aussi d'avancer qu'ils ne se considèrent pas encore des sujetslecteurs-enseignants, puisqu'ils sont en train de constituer leur propre rapport à la poésie : aucune remarque n'était en lien avec la didactique ou leur formation professionnelle, car aucune question n'était tournée vers les élèves ou vers leur pratique d'enseignement, par exemple : «Et si je devais enseigner vos poèmes, comme devrais-je m'y prendre? »

Enfin, malgré une consigne sans contrainte, les invitant à écrire un poème pour exprimer leur réaction vis-à-vis d'un poème de leur choix, la très grande majorité a écrit un poème «à la manière de ». Nous sommes portées à croire qu'ils ont fait ce choix, d'une part, pour s'approprier un langage nouveau, un style qui leur était initialement méconnu. Ce mimétisme langagier montre en effet que leurs compétences lectorales sont suffisamment aiguisées pour qu'ils soient en mesure d'analyser les caractéristiques d'un texte afin de les reproduire. Le Goff (2006, p. 253) l'observait aussi chez des lycéens choisissant l'écriture pastichante pour rendre compte de leur compréhension : «l'analyse textuelle opère nécessairement des prélèvements stylistiques ou génériques ciblés, en nombre limité, mais répété dans des proportions raisonnables pour accéder au statut de faits textuels exemplaires, suffisamment signifiants pour, une fois reproduits, créditer l'hypertexte de sa valeur de pastiche. Le rapprochement d'occurrences analogues permet d'établir ce que G. Genette appelle un «modèle de compétence » (Genette, 1982, p. 102). » D'autre part, le pastiche, en plus d'avoir pour finalité d'intérioriser un style, peut être dicté par l'admiration, ce que les étudiants peuvent aussi avoir voulu faire en imitant cette poète de la relève. Malgré tout, la présence du sujet est plutôt faible dans leur 
création - comme dans leur lecture du recueil. Manque d'expérience d'écriture poétique, faible fréquentation de textes poétiques, insécurités lectorales et créatrices, autant de facteurs pouvant expliquer cette absence du sujet vivant pleinement son rapport à la poésie. Mais ce sujet du poème, n'est-ce pas la finalité d'un enseignement de la poésie, comme le défendent Favriaud, Vinsonneau et Poletto (2017, p. 8) : «ce que devient un sujet, pour nous un élève (et probablement un maître), produisant ou lisant de la poésie et construisant ainsi dans la langue réinventée des poèmes un rapport toujours nouveau («amical », dirait le poète James Sacré) avec lui-même et avec l'altérité. » Comment les formateurs des enseignants et les didacticiens peuvent-ils arriver à former leurs étudiants en tant que sujets?

\section{Des pistes de formation initiale et continue des enseignants}

L'héritage de la tradition scolaire pèse lourd sur le rapport à la poésie des futurs enseignants et la reproduction des gestes de lecture ou d'écriture menés alors qu'ils étaient eux-mêmes élèves du secondaire est incontournable. Mais comment peut-on contrer ces effets du déterminisme et de la reproduction? Nous proposons deux pistes de formation, l'une pour la formation initiale et l'autre pour la formation continue, orientées de façon à ce qu'ils s'ouvrent à l'intranquillité de la poésie, pour reprendre l'expression de Pessoa (1999).

$1^{\circ}$

Pour transformer le rapport à la poésie des étudiants, pour ouvrir et complexifier leurs conceptions de cet objet, nous proposons de leur permettre de bonifier leur bibliothèque intérieure en y ajoutant des expériences poétiques variées et à les partager avec d'autres sujets lecteurs. Un cours disciplinaire de type «séminaire d'expériences littéraires », dans lequel les étudiants seraient appelés à lire un grand nombre d'œuvres littéraires de genres divers, dont autant de recueils de poésie que de romans et de pièces de théâtre, pourrait pouvoir s'insérer dans leur formation initiale. Cependant, l'évaluation des compétences lectorales au postsecondaire se fait souvent par des écrits très scolaires (analyse littéraire, dissertation, compte rendu critique...): les étudiants présentent ce qu'ils ont compris de l'œuvre et avancent quelques interprétations, appuyées de citations tirées du texte. Or, rendre compte de ses expériences littéraires peut prendre d'autres formes, dont celle des débats interprétatifs et de l'écriture créative.

Le débat interprétatif permet aux sujets-lecteurs de non seulement discuter de leurs diverses expériences de lecture, mais aussi de partager et de débattre oralement des interprétations qu'ils se font des textes littéraires (Dias-Chiaruttini, 2010; Sauvaire, 2013). Il s'agit d'un dispositif didactique encore peu présent dans les classes du secondaire (Aeby-Daghé, 2014), ce qui s'explique, notamment, par le manque de formation que les enseignants en ont reçue. Partant, mettre en place un tel dispositif dans des cours de littérature ou de didactique, dont notre proposition de «séminaire d'expériences littéraires », permettrait aux étudiants de non seulement discuter et de justifier leurs interprétations des textes, souvent difficiles à comprendre pour eux-mêmes, mais en outre de s'approprier les caractéristiques des genres lus, d'en saisir les défis qu'ils posent pour leur propre compréhension et pour celle d'élèves du secondaire, mais aussi de réfléchir aux expériences poétiques qu'ils peuvent générer et aux dispositifs didactiques à mettre en place pour les bonifier. 
Puis, pour permettre aux étudiants d'explorer autre chose que l'écriture d'imitation exercée au fil de leur scolarité, on pourrait les inviter à expérimenter et à pratiquer diverses écritures créatives pour se familiariser avec différents genres, styles, contraintes, processus de création, etc. Dans le cadre de ces ateliers d'écriture réalisés à la suite des débats interprétatifs, ils pourraient également être invités à participer à des «cercles de relectures » afin de discuter de leurs écrits, de commenter les créations de leurs collègues et de débattre des moyens créatifs et didactiques vers lesquels ils se tournent pour transformer leur processus de création et, plus largement, leur rapport à l'écrit... en vue de se constituer non seulement comme sujet lecteur/scripteur/créateur, mais aussi comme sujet-enseignants qui auront à former des élèves dans un ou des parcours du même ordre.

Un cours offrant un espace aux dimensions créatives des lectures et des écritures de la réception existe depuis 2007 à l'Université de Lausanne et cette innovation a eu des retombées intéressantes sur les étudiants, selon Florey et Cordonnier (2017). Même si un cours portant sur l'analyse de textes littéraires est certes offert dans quelques programmes de formation des enseignants québécois, aucune recherche n'a encore analysé les notions et corpus traités dans ces cours, la manière dont les cours sont donnés et les modes d'évaluation auxquels les étudiants sont soumis et qui, trop souvent, ont des effets sur leurs conceptions de l'objet enseigné. Pour la recherche, il est impératif que nous connaissions d'abord les objets enseignés et les pratiques d'enseignement dans les cours de didactique pour être ensuite en mesure de mettre en place des dispositifs didactiques pertinents et cohérents.

\section{$2^{\circ}$}

La formation initiale des enseignants ne représente toutefois, règle générale, que quatre années qui s'ajoutent à leur scolarité. Une fois dans l'exercice de leur profession, confrontés aux réalités du milieu (classes hétérogènes, tâches multiples, manque de temps, poids des évaluations périodiques, etc.), il se peut que de telles approches didactiques novatrices enseignées à l'université leur échappent dans le tourbillon du quotidien professionnel. Nous pensons qu'une formation continue du corps enseignant volontaire, répartie sur plusieurs années, et portant sur différents objets d'enseignement, dont la poésie, permettrait à ces derniers de recevoir un accompagnement qui les encouragerait à lire des recueils de poésie, à réfléchir à leur rapport à la poésie ainsi qu'aux relations de la poésie avec d'autres objets d'enseignement et différentes pratiques langagières, à découvrir des poètes qu'ils méconnaissent et à vivre - et partager - des expériences poétiques variées. Or, nous avons montré ailleurs que les enseignants disent manquer de temps pour lire en raison de leurs lourdes tâches de travail (Émery-Bruneau, 2014). Mais si l'une des tâches des enseignants de français du secondaire est d'enseigner des œuvres littéraires, alors comment peuvent-ils le faire s'ils n'ont pas le temps de lire des œuvres variées et pour faire des choix satisfaisants? Il s'agirait donc, pour mettre en œuvre une formation continue pérenne, de dégager dans leur horaire de travail actuel une plage horaire d'une demi-journée (3h) par semaine, soit le temps équivalent à un cours universitaire de 3 crédits. Ce temps dégagé pour la formation continue leur permettrait de 
lire des œuvres littéraires, d'en discuter, d'être accompagnés par des formateurs, chaque semaine, de façon encadrée et soutenue ${ }^{4}$.

\section{Conclusion}

Dans les travaux sur les théories de la réception, il a été montré que la créativité du lecteur (la création du texte de lecteur) trouve ses origines dans ses expériences personnelles de lecture et résulte d'un tissage entre le texte de l'auteur et la vie du lecteur : «Écrire sa lecture engage une image de soi » (Rouxel, 2017, p. 149). Or, il semble que la place d'un sujet - singulier, complexe et toujours inscrit dans un rapport à l'autre et au monde - est peu présente à l'école, comme à l'université d'ailleurs : pour relever le pari de moins formater les étudiants en FIEF, c'est-à-dire de moins les former à (ré)agir scolairement, ouvrons-leur les voies pour qu'ils s'émancipent de la reproduction des pratiques qu'ils ont connues/vécues élèves. Nous avons la responsabilité de les amener à vivre des expériences poétiques variées, de leur donner de l'espace pour partager et débattre de leurs lectures créatives, de les outiller pour transformer leur rapport à la poésie et, plus largement, à la littérature. Pour la formation des enseignants, il nous semble incontournable de mieux cerner les finalités de l'enseignement de la poésie dans un contexte d'enseignement et d'apprentissage du français, de la scolarité obligatoire au postsecondaire. Nous y travaillerons dans une prochaine contribution.

\section{Références}

Aeby-Daghé, S. (2014). Candide, La fée carabine et les autres, vers un modèle didactique de la lecture littéraire. Bruxelles : Peter Lang.

Bardin, L. (1993). L'analyse de contenu, Paris : PUF.

Bayard, P. (1998). Qui a tué Roger Ackroyd, Paris : Minuit.

Benveniste, É. (1969). Sémiologie de la langue. Semiotica, 1(1), 1-12.

Bonnefoy Y. (1998). Remarques sur l'enseignement de la poésie au lycée, in : E. Morin, \& Y. Bonnefoy (Eds.), Articuler les savoirs. L'enseignement de la poésie (pp. 106119). Paris : CNDP.

Bourdieu, P. (1979). La Distinction : critique social du jugement. Paris : Minuit.

Bourdieu, P. (1980). Le sens pratique. Paris : Éditions de Minuit.

Brillant-Rannou, N. (2010). Le lecteur et son poème. Lire en Poésie : expérience littéraire et enjeux pour l'enseignement du français en lycée (Thèse de doctorat, Université Rennes 2).

Brillant-Rannou, N. (2016). Le recueil à quatre mains et la lecture dialoguée dans les marges : conception et expérimentation de deux dispositifs de lecture subjective de poésie, in : N. Brillant-Rannou, C. Boutevin, \& M. Brunel (Eds.), Etre et devenir lecteur(s) de poèmes. De la poésie patrimoniale au numérique (pp. 87-109).

Namur : Presses universitaires de Namur.

Chartrand, S.-G., \& Lord, M.-A. (2010). Pratiques déclarées d'enseignement de l'écriture et de la lecture. Québec français, 156, 30-31.

Chartrand, S.-G., Émery-Bruneau, J., \& Sénéchal, K. (2015). Caractéristiques de 50 genres pour développer les compétences langagières en français. Québec : Didactica, c.é.f.

\footnotetext{
${ }^{4}$ Mais il va de soi, certes, qu'une volonté politique est essentielle pour dégager des fonds afin que ce type de formation continue du corps enseignant puisse être mise en place au Québec.
} 
Collot, M. (2005). La poésie moderne et la structure d'horizon. Paris : PUF.

Dezutter, O., Larivière, I., Bergeron, M.-D., \& Morissette C. (2007). Les pratiques déclarées des enseignants québécois dans la sélection et l'exploitation des œuvres complètes inscrites au programme de lecture des élèves, in : É. Falardeau, C. Fisher, C. Simard et N. Sorin, La didactique du français. Les voies actuelles de la recherche (pp. 83-100). Québec : Presses de l'Université Laval.

Dias-Chiaruttini, A. (2010). Le débat interprétatif dans l'enseignement de la lecture et de la littérature à l'école (Thèse de doctorat, Université Lille 3 -Charles de Gaulle).

Dufays, J.-L. (1994). Stéréotypes et lecture. Liège : Mardaga.

Duval, I. (2004). La poésie et l'enseignement du français. Québec français, 135, 42-45.

Duval, I., \& Turcotte, M. (2007). Dynamiser l'enseignement de la poésie contemporaine. Québec français, 147, 62-64.

Duval, I. (2014). Le ciel comme passage. Montréal : les Herbes rouges.

Émery-Bruneau, J. (2010). Le rapport à la lecture littéraire. Des pratiques et des conceptions de sujets-lecteurs en formation à l'enseignement du français à des intentions didactiques (Thèse de doctorat, Université Laval).

Émery-Bruneau, J. (2013). La formation à l'enseignement des textes littéraires au secondaire québécois : entre reproduire des pratiques et transformer la dimension praxéologique du rapport à la lecture littéraire. Actes de la Biennale internationale de l'éducation de la formation et des pratiques professionnelles, Paris.

Émery-Bruneau, J. (2014). La littérature au secondaire québécois : conceptions d'enseignants et pratiques déclarées en classe de français. Revue de linguistique et de didactique des langues, 49, 71-91

Émery-Bruneau, J. (2016). Comprendre l'action didactique par l'analyse des objets enseignés : l'exemple de deux séquences d'enseignement de la littérature au secondaire. Nouveaux cahiers de la recherche en éducation, 18(2), 115-137.

Favriaud, M., Vinsonneau, M., \& Poletto, M. (2017). Les Chemins de poésie d'Alep. Poétique et didactique du dire-lire-écrire à l'école primaire. Limoges : LambertLucas.

Florey, S., \& Cordonnier, N. (2017). Savants encore austères et amoureux vraiment fervents? Examen parallèle de deux écritures de la réception à l'université, in : $\mathrm{F}$. Le Goff \& M.-J. Fourtanier (Eds.), Les formes plurielles des écritures de la réception. Volume 1 : genres, espaces et formes (pp. 185-199). Namur : Presses universitaires de Namur.

Genette, G. (1982). Palimpsestes. Paris : Seuil.

Langlade, G., \& Fourtanier, M.-J. (2007). La question du sujet lecteur en didactique de la lecture littéraire, in : E. Falardeau, C. Fisher, C. Simard \& N. Sorin, La didactique du français. Les voies actuelles de la recherche (pp. 101-123). Québec : Presses de l'Université Laval.

Langlade, G. (2012). Chartreuse(s) de Parme : d'une lecture subjective à l'autre. Recherches \& Travaux, 83, 41-53.

L'Écuyer, R. (1990). Méthodologie de l'analyse développementale des contenus. Méthode GPS et concept de soi. Québec : Presses de l'Université du Québec.

Le Goff, F. (2006). Écriture d'invention, réécriture et enseignement de la littérature (Thèse de doctorat, Université de Lorraine).

Louichon, B. (2017). La réception scolaire des œuvres patrimoniales ou les Objets 
Sémiotiques Secondaires à l'école, in F. Le Goff \& M.-J. Fourtanier (Eds.), Les formes plurielles des écritures de la réception. Volume $1:$ genres, espaces et formes (pp. 19-33). Namur : Presses universitaires de Namur.

Mauron, C. (1963). Des métaphores obsédantes au mythe personnel : introduction à la psychocritique. Paris : éd. José Corti.

MÉQ (2005). Programme de formation de l'école québécoise. Enseignement secondaire, premier cycle. Québec : Gouvernement du Québec.

MÉLS (2009). Programme de français, langue d'enseignement, du deuxième cycle du secondaire. Québec : Gouvernement du Québec.

MÉLS (2011). Progression des apprentissages en français, langue d'enseignement. Québec : Gouvernement du Québec.

Melançon, J., Moisan, C.. \& Roy, M. (1988). Le discours d'une didactique. La formation littéraire dans l'enseignement classique au Québec (1852-1967). Québec : Nuit blanche éditeur.

Meschonnic, H. (2017). Infiniment à venir suivi de Pour le poème et par le poème. ParisOrbey: Arfuyen.

Pessoa, F. (1999). Le Livre de l'intranquillité. Paris : éd. Christian Bourgeois.

Picard, M. (1986). La lecture comme jeu. Paris : Éditions Minuit.

Rouxel, A. (2017). Créativité de la réception : de l'investissement subjectif au projet artistique, in : F. Le Goff \& M.-J. Fourtanier (Eds.), Les formes plurielles des écritures de la réception. Volume 1 : genres, espaces et formes (pp. 147-168). Namur : Presses universitaires de Namur.

Saint-Gelais, R. (2011). Fictions transfuges. La Transfictionnalité et ses enjeux. Paris : Seuil.

Sauvaire, M. (2013). Diversité des lectures littéraires : comment former des sujets lecteurs divers? (Thèse de doctorat, Université Laval et Université de Toulouse 2-Le Mirail).

Siméon, J.-P. (2015). La poésie sauvera le monde. Paris : Le Passeur.

Tauveron, C. (1999). Comprendre et interpréter le littéraire à l'école : du texte réticent au texte proliférant. Repères, 19, 9-38. 
Biographie des auteures

Judith Émery-Bruneau est professeure à l'Université du Québec en Outaouais (Gatineau). Ses domaines de recherche s'inscrivent dans le champ de la didactique du français et portent sur l'enseignement et l'apprentissage de la littérature. Elle s'intéresse de près aux pratiques d'enseignement de la littérature et de la poésie au secondaire, à la progression des lecteurs aux différents niveaux de la scolarité obligatoire, à la didactisation de pratiques sociales comme le slam et la performance poétique, ainsi qu'au rapport à la lecture littéraire d'étudiants en formation initiale à l'enseignement. Elle fait partie de deux regroupements de chercheurs: l'Équipe de Recherche Littératie et Inclusion (ÉRLI) et le Centre de recherche interuniversitaire sur la formation et la profession enseignante (CRIFPE).

Camille S. Leclerc est étudiante à la maitrise à l'Université du Québec en Outaouais (Gatineau), sous la direction de Judith Émery-Bruneau. Elle s'intéresse au rôle des ressources subjectives et des modes opératoires des sujets lecteurs dans la validation d'interprétations intersubjectives de poèmes au sein de débats interprétatifs. 